\title{
Système Empress et restaurations prothétiques
}

\section{RÉSUMÉ}

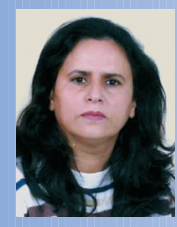

Amal EL YAMANI

Professeur agrégé,

Service de prothèse fixée,

Faculté de médecine dentaire.

Rabat - Maroc.

Villa 15 bassatine El menzeh,

route des Zaers,

Rabat - Maroc.

\section{Hicham SOUALHI}

Chirurgien-dentiste,

Spécialiste en prothèse fixée,

Service de prothèse fixée,

Faculté de médecine dentaire,

Rabat - Maroc.

\section{Jamal EL BERNOUSSI}

Professeur d'enseignement supérieur,

Service de prothèse fixée,

Faculté de médecine dentaire,

Rabat - Maroc
Les techniques et les matériaux permettant la réalisation de prothèses sans infrastructures métalliques se multiplient. Parmi eux le système Empress permet d'allier un bon résultat esthétique à de bonnes propriétés mécaniques et une mise en œuvre simple au laboratoire.

L'évolution des techniques de laboratoire associée au progrès dans le domaine d'adhésion permet d'indiquer ce procédé pour la réalisation des coiffes, facettes ou inlays sans support en métal, ayant une translucidité et un rendu esthétique les plus proches possibles de la dent naturelle.

Le succès de ces restaurations dépend outre de la préparation dentaire, d'un respect rigoureux du procédé d'élaboration au laboratoire.
- Empress

- restaurations prothétiques

- protocole de laboratoire 


\section{Introduction}

$>$

Depuis une vingtaine d'années trois objectifs préoccupent les cliniciens : plus d'esthétique, une biocompatibilité accrue et un souci d'économie tissulaire.

En effet, la prothèse céramo-métallique a longtemps constitué la synthèse, la plus cliniquement acceptable, entre «beauté» et "solidité». Mais la présence d'une infrastructure métallique particulièrement au secteur antérieur constitue une barrière à la circulation de la lumière et contribue à assombrir les tissus gingivaux environnants $[1,11]$. C'est ainsi, que plusieurs procédés tout en céramique ont été mis au point dans le but de remplacer l'armature métallique des restaurations prothétiques.
Au cours de ces dix dernières années, en plus du développement de nouveaux systèmes céramo-céramiques, le perfectionnement du collage et des traitements de surface permet d'envisager plus sereinement ce type de restauration qui offre translucidité et biocompatibilité.

Après le succès des restaurations unitaires entièrement en céramique, il a été logique que I'ensemble des systèmes ait été amélioré pour étendre leurs indications aux différents types de restaurations prothétiques.

Parmi ces systèmes nous nous sommes intéressés au système Empress et à son apport aux restaurations prothétiques.

\section{Avantages des restaurations en céramique}

- Bon rendu esthétique en améliorant la profondeur de translucidité ainsi que la transmission lumineuse de la restauration.

- Biocompatibilité par l'absence de toute structure métallique[11].

- Haute résistance aux sollicitations fonctionnelles.

- Possibilité de sceller ou de coller.

\section{Le système Empress}

De point de vue historique le système Empress a été développé par la firme Ivoclar en 1983 et présenté à la profession en 1990.

Comme pour tout système entièrement en céramique nous décrirons un matériau et une technique d'élaboration.
- Bonne tolérance parodontale : d'une part la qualité d'adaptation est à un niveau semblable à celle des restaurations céramométalliques, ce qui assure une bonne qualité du joint périphérique, d'autre part I'utilisation de matériau mimétique évite l'enfouissement systématique des limites cervicales dans le sillon gingivo-dentaire[20].

\section{Le système Empress : matériau et composition}

Initialement, dans la technique Empress $\left.\right|^{\circledR}$ on utilisait un verre renforcé par la leucite; dans la technique Empress II ${ }^{\circledR}$, L'IPS Empress 2 se 
compose d'un matériau de disilicate de lithium $\left(\mathrm{SiO}^{2}-\mathrm{Li}^{2} \mathrm{O}\right)$ qui servira pour la confection de I'armature. Ce matériau est disponible sous la forme de lingotins d'aggloméré verre-céramique. Les cristaux de disilicate de lithium représentent $60 \%$ du volume et leur disposition dans la masse vitreuse permet une dispersion lumineuse qui donne une réelle translucidité de la chape[1, 6].

L'armature est secondairement recouverte d'une céramique cosmétique dont le coefficient d'abrasion est très proche de celui des dents naturelles ce qui évite aux dents antagonistes d'être usées lors de la fonction.

\section{Technique}

La méthode de fabrication au laboratoire est très similaire à celle suivie pour les restaurations métalliques car elle utilise la méthode de la cire perdue[10, 13].

Ainsi les pièces prothétiques sont sculptées dans leurs formes définitives, ou sous forme $d$ 'infrastructures sur le modèle de travail avec une cire brûlant sans résidus pour éviter toute pollution de la céramique qui se traduirait par des colorations indésirables dans le matériau (fig. 1).

Elles sont fixées ensuite dans le sens axial par la zone incisale ou occlusale sur une tige de coulée (fig. 1). La mise en revêtement s'effectue dans un cylindre en papier avec un matériau réfractaire approprié (fig. 2).

Le bloc réfractaire est ensuite préchauffé dans un four à $850^{\circ} \mathrm{C}$ avec le lingotin de céramique et le piston, puis stabilisé 60 minutes (fig. 3 a, 3 b).

Ensuite le cylindre est positionné au centre du four de pressée, le lingotin introduit dans la cheminée du cylindre et le piston mis en place. La température s'élève alors jusqu'à $1075^{\circ} \mathrm{C}$ et le processus de pressée se déroule automatiquement (fig. 4).

La pièce pressée sera démoulée après refroidissement complet. Le réfractaire est découpé a l'aide de disques et de fraises. L'élément prothétique est ensuite dégagé $d u$ réfractaire par un sablage aux billes de verre de 50 à 100 microns, sous pression de 2 bars (fig. 5 a, 5 b).
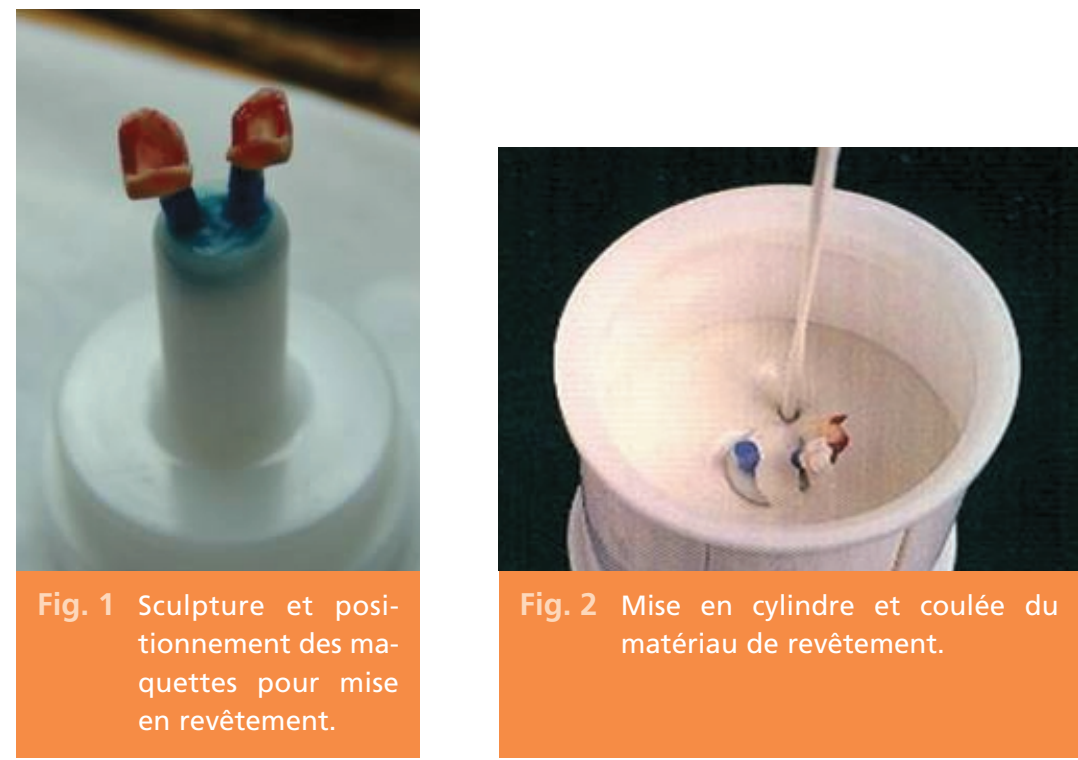

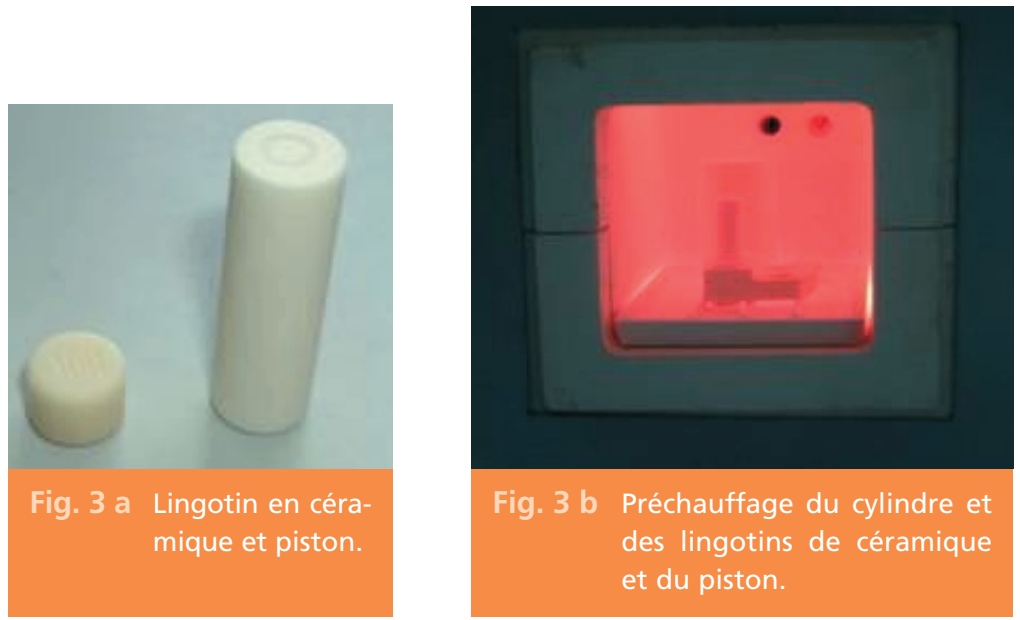

Fig. 3 b Préchauffage du cylindre et des lingotins de céramique et du piston.

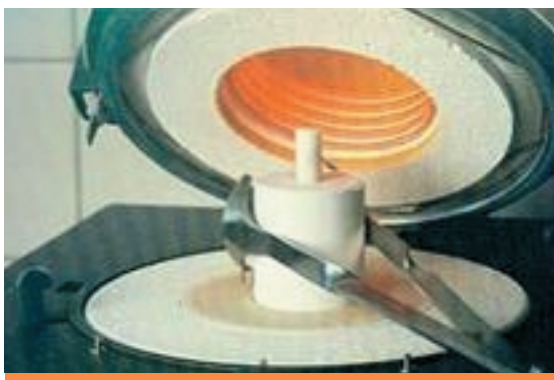

Fig. 4 Pressée de la céramique dans un four à pressée.

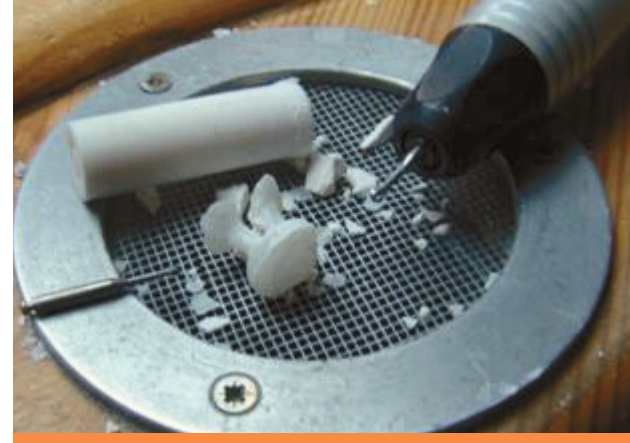

Fig. 5 a Démoulage des pièces prothétiques.

On peut utiliser deux techniques pour la caractérisation :

- technique par maquillage de surface: on réalise la totalité de la facette ou de la couronne en Empress ${ }^{\circledR}$, puis on la maquille en surface et on la glace (fig. 6 a, 6 b) ;

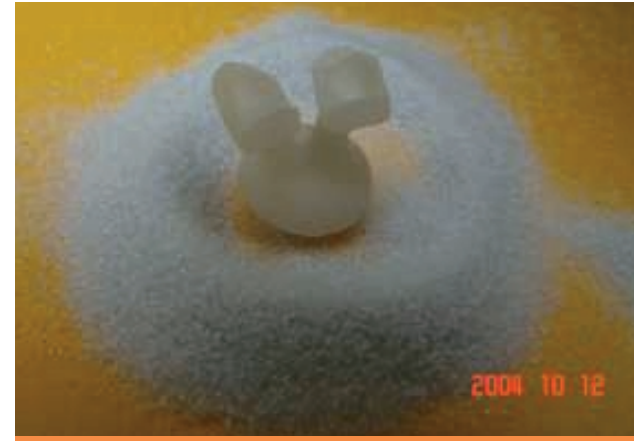

Fig. 5 b Sablage des pièces prothétiques.

- technique de stratification : on réalise par la même technique une infra-structure renforcée qui sera secondairement stratifiée par une céramique cosmétique choisie selon la teinte finale souhaitée. 

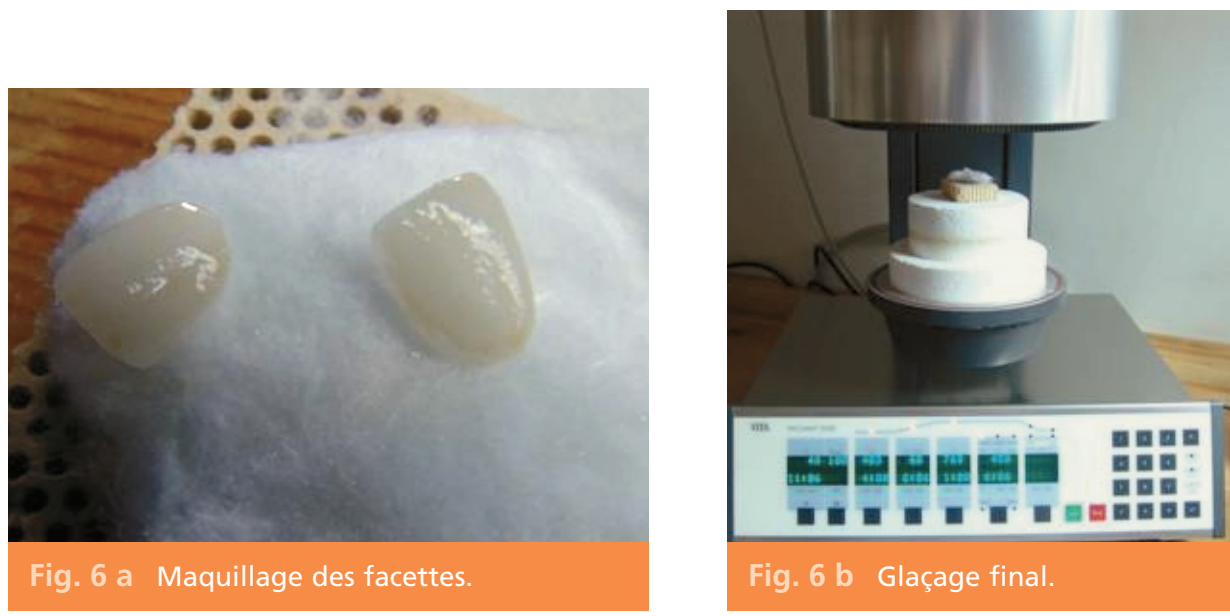

Fig. 6 b Glaçage final.

\section{Propriétés comparées avec les autres systèmes céramiques}

\section{Qualité mécanique}

Les céramiques contrairement aux métaux, sont des matériaux a rupture fragile, c'est- à-dire cassante sans déformation préalable. En fonction de leur constitution la force à mettre en jeu pour les rompre est plus ou

\section{Tableau I Propriétés mécaniques des différents systèmes céramiques.}

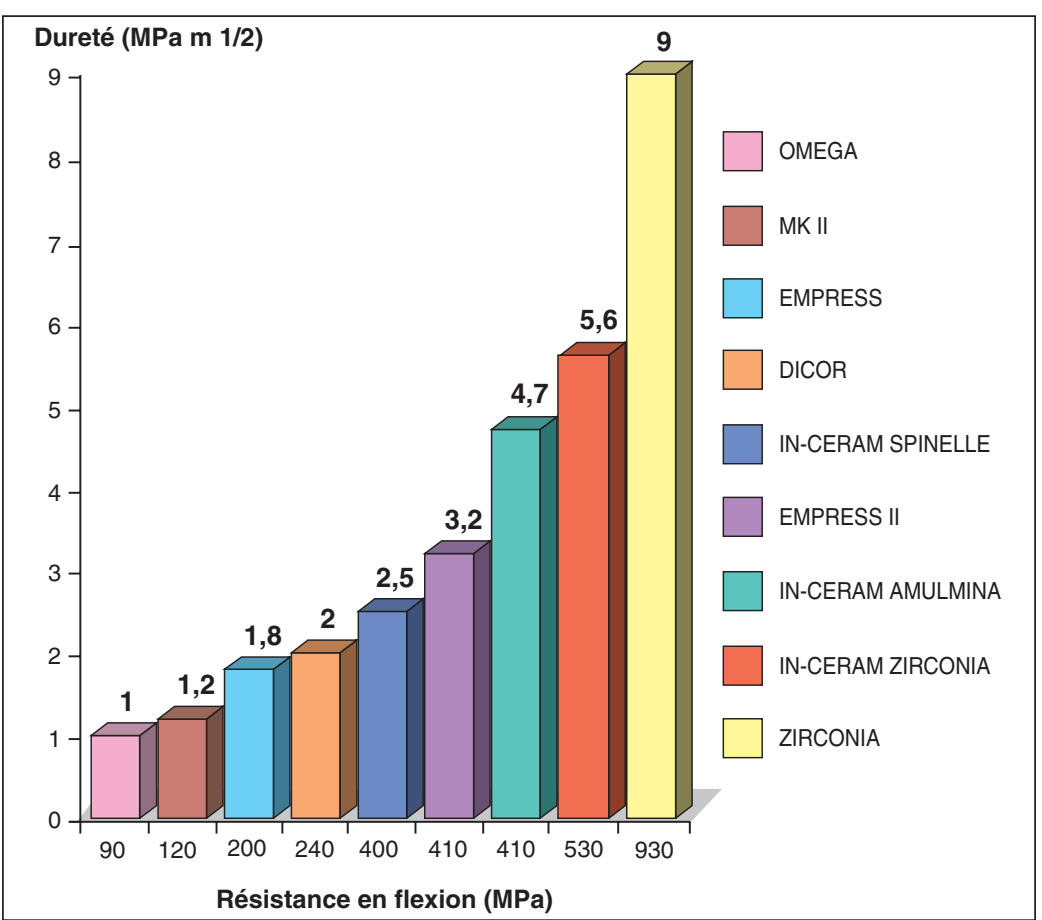


moins importante et la céramique est dite plus ou moins résistante. Leur résistance est directement liée à la diminution de la phase vitreuse fragile et à l'augmentation des charges[12,18]. Les céramiques injectées sous haute pression de type Empress I et Empress II possèdent une phase vitreuse importante enrobant les charges cristallines (leucite ou dissilicate de lithium). Les propriétés mécaniques qui en découlent sont ainsi médiocres par rapport aux autres systèmes.

Les propriétés mécaniques des matériaux de restauration "tout céramique» sont représentées dans le tableau I[5].

\section{Précision d'adaptation}

Dans une étude évaluant la qualité d'adaptation de trois céramiques[19] :

- empress 2 ;

- inceram celay ;

- inceram convent.

Les valeurs de joint obtenues en microns sont :

- empress $2: 46+-16$;

- inceram celay : $83+-33$;

- inceram convent : $112+-55$.

Ainsi il n'existe pas de différence d'adaptation majeure entre les différents systèmes céramocéramiques. Les valeurs du joint obtenues sont inférieures aux 120 microns considérés par Mac Lean comme cliniquement acceptables et durables.

\section{Qualités esthétiques}

La réflexion de la lumière et de même le caractère translucide ou opaque d'un système est directement liée au nombre et à la composition des charges dans la céramique.

Ainsi I'armature de I'Empress et de I'In-ceram spinelle sont translucides, celle de l'Alumine Procera est semi-translucide alors que I'Inceram Alumina et Zirconia gagnent en opacité avec la résistance mécanique[4, 12,15].

Une armature translucide est un avantage en cas de dents pulpées non dyschromiées, alors qu'un pilier dyscoloré nécessite une armature plus opaque.

\section{Qualité de la liaison} céramique-résine

ou possibilité

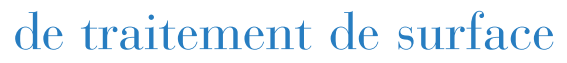

Les céramiques sont par nature des matériaux cassants et leur collage est un moyen efficace de pallier leur énergie emmagasinée dans la céramique au travers des tissus dentaires sous-jacents $[2,14]$.

Le matériau utilisé pour le système Empress fait partie des céramiques dites mordançables. Ce sont des céramiques qui contiennent en quantité plus ou moins importante de silicates. Cette phase vitreuse peut être mordancée par un acide fort, l'acide fluorhydrique permettant de créer un relief propice au collage[18].

Le mordançage doit être précédé par un sablage et suivi dans un second temps, par un silanage. Ce protocole permet d'obtenir des valeurs d'adhésion très importante. 


\section{Applications cliniques}

\section{Facettes}

(fig. 7 a à c)

L'évolution des systèmes de collage et des systèmes entièrement en céramique a permis d'élargir les indications des facettes en céramique, qui peuvent traiter de nombreuses situations cliniques en préservant la vitalité pulpaire.

Les facettes en céramique doivent principalement répondre a deux impératifs: qualités esthétiques optimales et maîtrise de la liaison colle/céramique.

L'expérience tirée des facettes en céramique conventionnelle feldspathique aux faibles qua- lités mécaniques a démontré que, grâce a la cohésion obtenue avec la structure dentaire résiduelle, la résistance du matériau n'est pas déterminante[2, 14].

Pour autant, il est aujourd'hui possible de tirer parti de l'amélioration des propriétés mécaniques des nouvelles céramiques ainsi que de leur translucidité tel le système Empress[12,16].

\section{Couronnes unitaires}

Sur les dents antérieures, tous les systèmes présentent des qualités mécaniques suffisantes pour supporter les charges subies par ces dents[10,16].
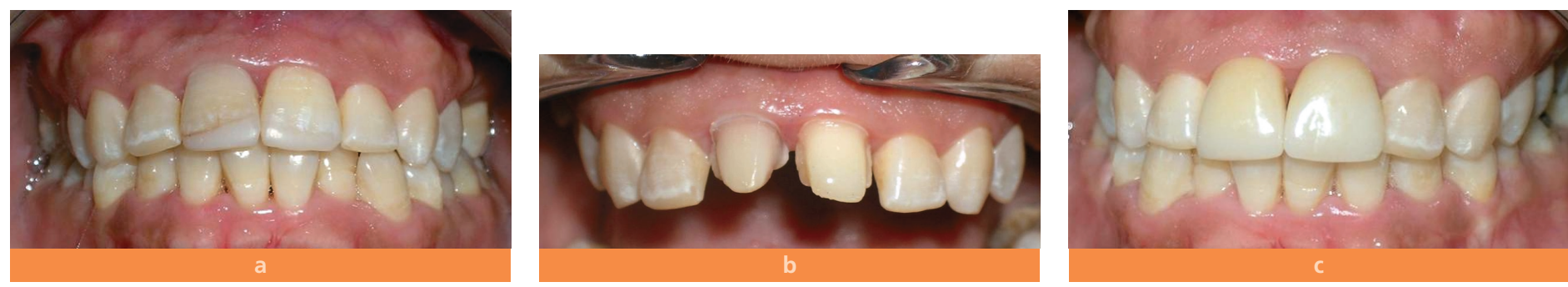

\section{Fig. 7 a à c Réalisation de facette en Empress ;}

a : cas initial : restaurations inesthétiques type IV au composite de la 11 et 21 ;

b : préparation pour facette avec retour palatin ;

c: résultat final.
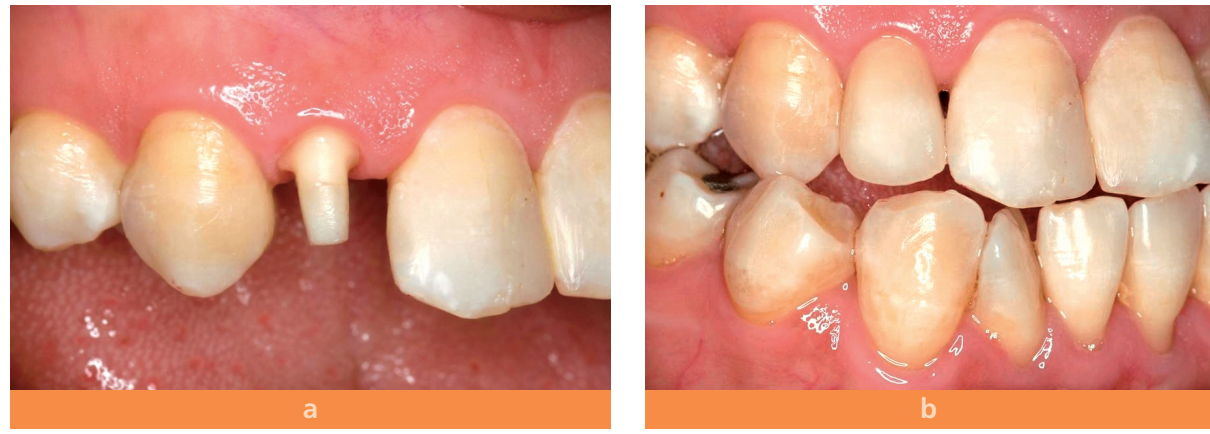

Fig. 8 a et b Couronne en Empress ;

a : la préparation de 12 ;

b : résultat après collage. 
La translucidité du système EMPRESS l'indique pour la restauration des dents dépulpées ou présentant des reconstitutions corono-radiculaires esthétiques non métalliques (fig. 8 a et b).

Au niveau des dents postérieures, les contraintes importantes propres a ce secteur sont en faveur de l'utilisation de systèmes plus résistants (In-ceram, Procéra)[15].

\section{Inlays-onlays}

(fig. $9 \mathrm{a}$ à c)

Pour les inlays-onlays, I'utilisation de matériaux résistants est en faveur des céramiques alumineuses.

Cependant, l'évolution du système Empress vers une vitro-céramique plus résistante
(Empress II), associée à la simplicité de sa mise en œuvre et sa qualité d'adhérence l'indiquent pour ce type de restauration[7, 11].

Toutefois, les céramiques restent concurrencés par les composites de laboratoire[8].

\section{Bridges}

(fig. 10 et b)

Le système Empress $\|^{\circledR}$ présente l'inconvénient d'utiliser un matériau dont la résistance mécanique est la plus faible par rapport aux autres systèmes (système Procera ${ }^{\circledR}$, système InCeram $^{\circledR}$ ). II est d'ailleurs contre-indiqué pour les bridges postérieurs[3, 4].

La réalisation des bridges par le système IPS Empress 2 se limite au remplacement d'une dent antérieure en avant de la deuxième pré-
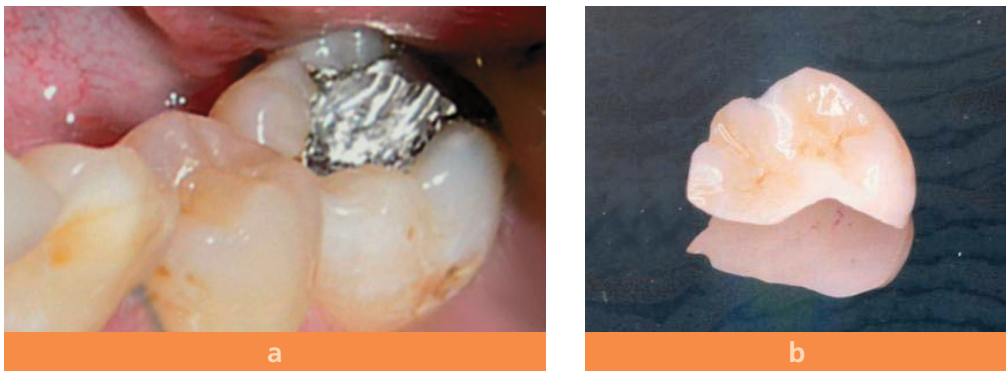

Fig. 9 a à c Réalisation d'un inlay en Empress ;

a : amalgame occluso-distale sur la 36 ;

b : inlay en Empress ;

c: résultat final.
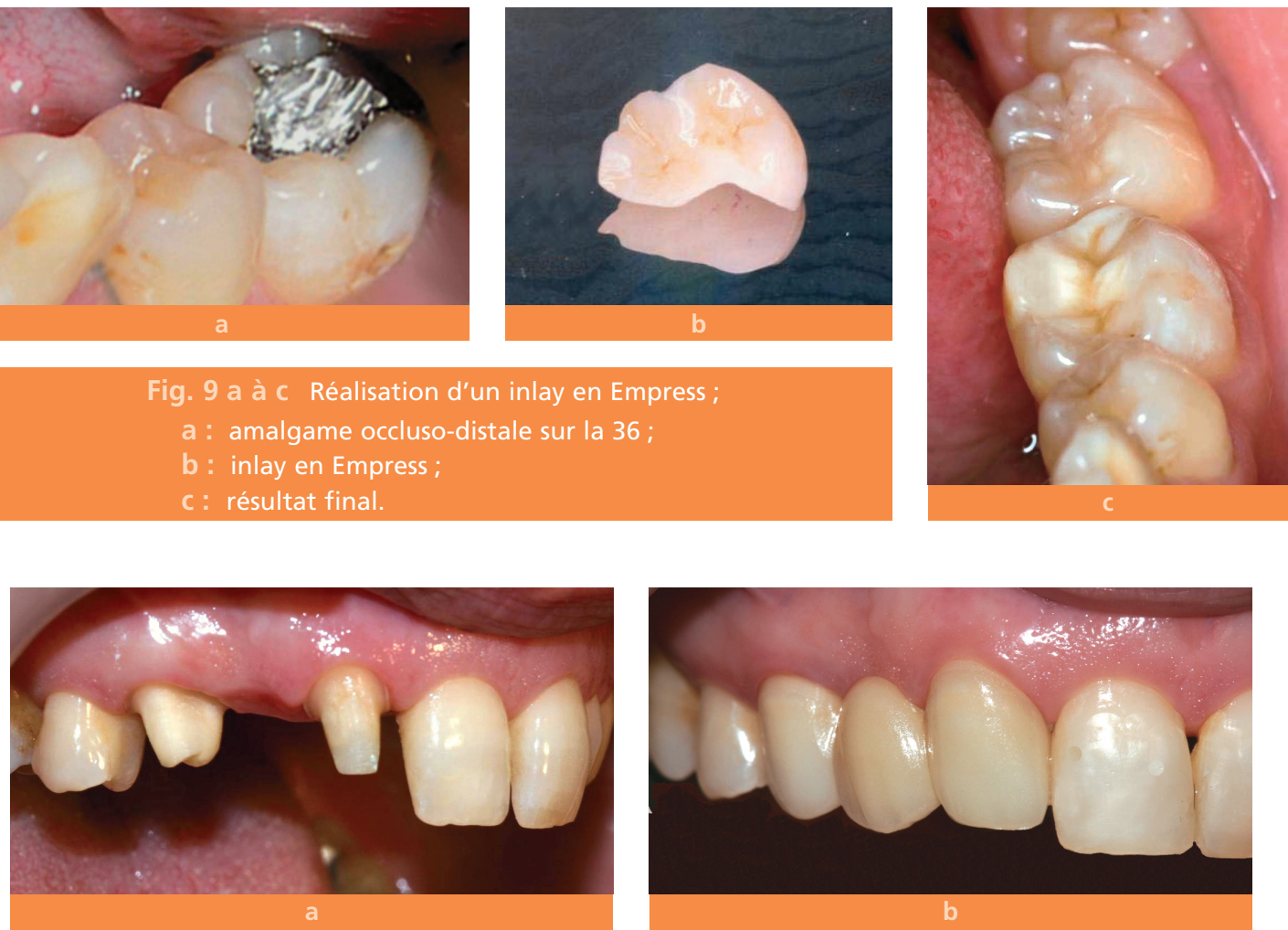

Fig. 10 a et $b$ Bridge en Empress ;

a : préparation de 12 et 14 pour recevoir un bridge en Empress ;

b : résultat final. 
molaire, avec une travée ne dépassant pas $10 \mathrm{~mm}$. La largeur de l'intermédiaire de bridge ne devra pas excéder $11 \mathrm{~mm}$ antérieurement à la canine et $9 \mathrm{~mm}$ postérieurement à la canine. Ces mesures sont faites entre les surfaces proximales des dents piliers non préparées, en regard de l'emplacement du pontique[4].

\section{Reconstitutions}

\section{corono-radiculaires}

Le caractère foncé et opaque des tenons métalliques nuit à la propagation de la lumière et confère aux tissus durs et mous environnants un aspect sombre et grisâtre en particulier lorsque le parodonte est fin. Ainsi, afin d'optimiser l'esthétique des restaurations entièrement en céramique, des reconstitutions corono-radiculaires esthétiques ont été proposées.
Ces reconstitutions corono-radiculaires reposent sur I'utilisation de tenons préfabriqués englobés dans la céramique. Une possibilité consistait à claveter une reconstitution en alumine-zircone à $50 \%$ dans la racine des dents par des tenons calibrés soit en fibres de verre ou de silice de quartz.

Plus récemment, la société Ivoclar a commercialisé un nouveau tenon endodontique en oxyde de zirconium, le Cosmopost ${ }^{\circledR}$. Une céramique contenant de l'oxyde de zirconium (lingotin IPS Empress Cosmo) peut être pressée et adaptée par-dessus (fig. 11 a et b)

Ce type de reconstitution est extrêmes rigide et peut transmettre les forces occlusales à la racine du fait qu'il n'offre aucune possibilité d'absorption des contraintes[9]. II est donc conseillé de le réserver aux dents qui ont des structures radiculaires suffisantes.
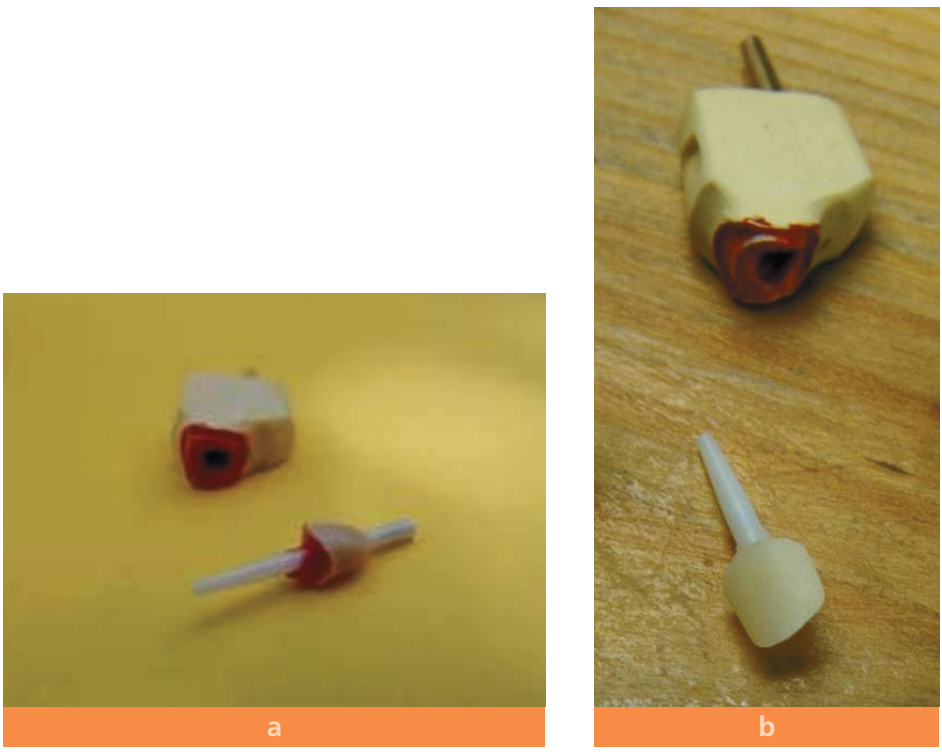

Fig. 11 a et $b$ Inlays core en ceramique IPS Empress pressé sur un tenon en oxyde de zirconium (Cosmopost ${ }^{\circledR}$ ) ;

a : montage de la cire autour du tenon ;

b : inlay core après la presse de la céramique. 


\section{Collage}

La nouvelle génération Empress II contrairement à la précédente autorise aussi bien le collage que le scellement des restaurations avec un ciment verre ionomère modifié par adjonction de résine[4].

Néanmoins la plupart des auteurs s'accordent sur le rôle du collage dans l'amélioration des qualités mécaniques de la céramique[2].

Un traitement de surface de la céramique sera cependant indispensable, il associera :

- sablage à l'oxyde d'alumine 100 microns ;

- mordançage à l'acide fluorhydrique 2,5-10 \% pendant 20-30 secondes :

- neutralisation de l'acide par lavage avec de l'eau mélangée à une poudre neutralisante composée de carbonate de calcium et de car-

\section{Conclusion}

La demande en matière d'esthétique de nos patients oblige le praticien à rechercher de plus en plus des techniques de restaurations capables de redonner un aspect naturel à la denture.

Ainsi depuis une quinzaine d'années, le matériau Empress II permet des restaurations esthétiques estimées être parmi les meilleures comparativement aux autres matériaux «tout céramique». Ce matériau permet la réalisation des coiffes, facettes ou inlays sans support bonate de sodium (IPS Ceramic poudre neutralisante", Ivoclar-Vivadent) ;

- deux applications successives de silane (Monobond S", Ivoclar-Vivadent) laissées à l'évaporation pendant 60 secondes chacune ; -enfin, application uniforme d'une fine couche d'adhésif amélo-dentinaire (Excite", Ivoclar-Vivadent) sans photopolymérisation.

Selon l'étude d'Alfredo Meyer I'application d'un agent silanique est l'élément le plus déterminant dans la qualité de la liaison résine céramique, plus que le mordançage à l'acide fluorhydrique ou le sablage[14].

En bouche, la préparation des dents piliers est identique pour tous les systèmes céramiques.

métallique, ayant une translucidité et un rendu esthétique le plus proche possible de la dent naturelle.

L'évolution des techniques de laboratoire, du perfectionnement de l'adhésion amélaire et surtout dentinaire et de la mise au point de nouveaux composites de collage, ont permis de codifier de façon stricte les protocoles cliniques et de laboratoire pour que ce système puisse s'imposer dans notre pratique courante. 


\section{Bibliographie}

1. Allard $\mathrm{Y}$.

Une nouvelle céramique:

IPS Empress.

Réalités Cliniques

1991;2(4):477-488.

2. Bartala M.

Scellement ou collage?

Le choix raisonné.

Cahiers de Prothèse

2002;117:67-81.

3. Brochu J-F, El-Mowafy O. Longevity

and Clinical Performance

of IPS-Empress

Ceramic Restorations:

A Literature Review.

J Can Dent Assoc

2002;68(4):233-7.

4. Carré J, Nardari C.

Une nouvelle

conception d'armature.

Bridge tout céramique

I PS Empress 2.

Proth Dent 1999;152:21-25.

5. Guastalla O, Viennot S,

Allard Y.

Collages

en odontologie.

EMC-Ondontologie

2005;1:193-201.

6. De Rouffignac M,

Decooman J.

IPS Empress 2 (Ivoclar).

Synergie Proth

2003;2(2):127-131.

7. Fleiter B, Tirlet G.

Inlays,

onlays de céramique

collés.

Art Tech Dent

1991;2(6):429-436.

Synergie prothétique

2000:2 (2):127-138.

8. Garber D, Goldstein R. Inlays et onlays en céramique

et en composite.

Restaurations postérieures

esthétiques.

Paris : CDP, 1995.

9. Gonthier $S$,

Desreumaux-Gonthier M.

Influence esthétique

des reconstitutions

corono-radiculaires.

Réalités cliniques

2003:14(4):443-456

10. Gormezo A.

La couronne

céramo-céramique

unitaire

antérieure.

Clinic 2004;25(3):135-146.

11. Laborde $\mathrm{G}$, lacrois $\mathrm{Ph}$, Margosian $\mathrm{P}$, Laurent $\mathrm{M}$.

Les systèmes

céramo-céramique.

Réalités cliniques

2004;15(1):89-104.

12. Laurent $\mathrm{M}$

Aboudharam $\mathrm{G}$

Laplanche O, Laborde G.

Céramique

sans armature métallique :

quels procédés

pour quelles indications ?

Cahiers de Prothèse

2002;119:7-15.

13. Mahiat $Y$.

Les facettes

en céramique :

4 technique

de réalisation

au laboratoire.

Statégie prothétique 1999;1(1):46-57.

14. Markus B, Avishani B Sadan, B, Matthias K. Resin-ceramic bonding: a review of the literature.

The Journal of Prosthetic

Dentistry March

2003;89(3):268-274.

15. McLean JW.

High-alumina ceramics

for bridge

pontic construction.

Br Dent J 1967;123:571-7.

16. Miara P, Touati B.

Principes cliniques

et de laboratoire

des facettes

en céramique

IPS EMPRESS

pour incisives inférieures.

Information dentaire,

n 4 du 27 janvier 1994.

17. Mauny F, Sadoun $M$,

Daniel $X$.

Les préparations coronaires

périphériques

pour procédés

céramo-céramiques.

Real Clin 1996;7:459-70.

18. Poujade J.-M, Zerbib C, Serre D.

Céramiques dentaires.

EMC-Dentisterie

2004;1:101-117.

19. Sorensen JA, Cruz M,

Mito WT.

Évaluation clinique

du nouveau système

vitrocéramique

IPS Empress 2

avec armature

en disilicate de lithium.

Signature Internationale 1999:4:114-10.

20. Wohlwend A, Scharer P.

Céramique sans métal

pour couronne,

inlays et facettes.

ATD 1992;3(1):15-21. 


\section{SUMMARY}

\section{Empress System and prosthetic restorations}

Amal EL YAMANI

Hicham SOUALHI

Jamal EL BERNOUSSI

\section{Keywords}

- Empress - prosthetic restorations - laboratory protocol
The techniques and the materials allowing the prosthesis realization without metal infrastructures are numerous. Among them the Empress system makes it possible to combine a good aesthetic result with good mechanical properties and a simple implementation at the laboratory.

Developments of laboratory technology associated with progress in the field with adhesion make it possible to indicate this process for the realization of the caps, facets or inlays without metal support, having a translucent and an aesthetic made nearest to the natural tooth.

The success of these restorations depends, in addition to dental preparation; on a rigorous respect of the laboratory processes.

\section{Questions - Réponses}

\section{1/ Réponse par vrai ou faux}

Les reconstitutions céramo-céramiques sont indiquées dans toutes les situations cliniques

Vrai $\square \quad$ Faux

\section{2/ QCM à une seule réponse}

Les RCR esthétiques foulées sont indiquées :

a - en cas de limite sous-gingivale

b - sous une couronne céramo-métallique

c - sous couronne céramo-céramique

d - une présence d'une seule paroi coronaire 